\title{
Analysis of Statoliths Displacement in Chara Rhizoids for Validating the Microgravity-Simulation Quality of Clinorotation Modes
}

\author{
Lars Krause $^{1,2,3} \cdot$ Markus Braun $^{2,4} \cdot$ Jens Hauslage ${ }^{1}$ (i) $\cdot$ Ruth Hemmersbach $^{1}$
}

Received: 25 October 2017 / Accepted: 5 December 2017 / Published online: 13 January 2018

(C) The Author(s) 2018. This article is an open access publication

\begin{abstract}
In single-celled rhizoids of the green algae Chara, positively gravitropic growth is governed by statoliths kept in a dynamically stable position 10-25 $\mu \mathrm{m}$ above the cell tip by a complex interaction of gravity and actomyosin forces. Any deviation of the tube-like cells from the tip-downward orientation causes statoliths to sediment onto the gravisensitive subapical cell flank which initiates a gravitropic curvature response. Microgravity experiments have shown that abolishing the net tip-directed gravity force results in an actomyosin-mediated axial displacement of statoliths away from the cell tip. The present study was performed to critically assess the quality of microgravity simulation provided by different operational modes of a Random Positioning Machine (RPM) running with one axis (2D mode) or two axes (3D mode) and different rotational speeds (2D), speed ranges and directions (3D). The effects of 2D and 3D rotation were compared with data from experiments in real microgravity conditions (MAXUS sounding rocket missions). Rotational speeds in the range of 60-85 $\mathrm{rpm}$ in 2D and 3D modes resulted in a similar kinetics of statolith displacement as compared to real microgravity data, while slower clinorotation (2-11 rpm) caused a reduced axial displacement and a more dispersed arrangement of statoliths closer to the cell tip. Increasing the complexity of rotation by adding a second rotation axis in case of 3D clinorotation did not increase the quality of microgravity simulation, however, increased side effects such as the level of vibrations resulting in a more dispersed arrangement of statoliths. In conclusion, fast 2D clinorotation provides the most appropriate microgravity simulation for investigating the graviperception mechanism in Chara rhizoids, whereas slower clinorotation speeds and rotating samples around two axes do not improve the quality of microgravity simulation.
\end{abstract}

Keywords Chara $\cdot$ Clinorotation $\cdot$ Gravitropism $\cdot$ Microgravity $\cdot$ Random positing machine $\cdot$ Sounding rockets

Jens Hauslage

jens.hauslage@dlr.de

1 Institute of Aerospace Medicine, Gravitational Biology, German Aerospace Center (DLR), Linder Hoehe, 51147 Cologne, Germany

2 Institute of Molecular Physiology and Biotechnology of Plants (IMBIO), Gravitational Biology, University of Bonn, Kirschallee 1, 53115 Bonn, Germany

3 Agrosphere (IBG-3) Institute of Bio- and Geosciences, Forschungszentrum Jülich GmbH, Wilhelm Johnen Straße, 52425 Jülich, Germany

4 Space Administration, German Aerospace Center (DLR), Koenigswinterer Str. 522-524, 53227 Bonn, Germany

\section{Introduction}

The International Space Station (ISS), satellites, sounding rockets, parabolic plane flights and drop towers are research facilities that provide years, month, minutes or only seconds of a real-microgravity $(\mu g)$ environment that has been intensively used in the last decades for gravitational biology research. However, experiments in real $\mu g$ are scarce, costly and time consuming. Therefore, different $\mu g$ simulation facilities like clinostats, Random Positioning Machine (RPM), rotating wall vessels and magnetic levitation are frequently used as attractive alternatives for complementing and preparing real-microgravity experiments in the 
near-Earth orbit. Furthermore, they enable with some limitations, such as sample diameter, biological studies of the impact of altered gravity conditions on Earth (Herranz et al. 2013; Brungs et al. 2016).

Clinostat capabilities include operation modes with one axis (2D clinostats) and two axes (3D clinostats). The classical 2D clinostat usually rotates constantly and slowly at $1-10$ revolutions per minute (rpm), whereas fast rotating clinostats (Briegleb 1992; Herranz et al. 2013) run at a selected speed between 60-90 rpm. Due to the physical constraints only small samples placed precisely along the rotation axis will experience acceleration forces which are thought to be below the threshold of the biological system. Specimen larger than a few millimeters or samples not placed very close to the center of the rotation axis will experience centrifugal forces in the periphery.

3D-clinostats and RPMs operate based on the same principle with two independently rotating frames mounted in a gimbal manner allowing complex movements to average the influence of the gravity vector (Hoson et al. 1997; van Loon 2007; Borst and van Loon 2009). In 3D real random mode, the RPM operates with a range of velocities and also the direction of rotation is changed in a real random manner determined by dedicated algorithms. The movement of the samples is randomly generated to avoid a repetitive pattern to which the test samples may adapt to.

The various modes and angular rotation velocities create specific sets of forces and respective side effects like stress and shear forces with different impacts as well as positive and negative accelerations on cells or biological organisms. Due to the fact that reports on clinostat and random positioning experimentation frequently do not adequately state the operational modes and precise physical parameters of the treatment (Hammond and Allen 2011), a comparison of experiment results, calculation of forces and determination of threshold values is in many cases very difficult hampering generalization of conclusions (Herranz et al. 2013).

A diversity of model organisms such as Arabidopsis thaliana seedlings and plant cell cultures, characean rhizoids, human immune cells and protists have been investigated by several European science teams on various microgravity simulation facilities and the results were compared with real $\mu g$ data (Herranz et al. 2013; Brungs et al. 2016). It became evident that a thoroughly performed assessment of side effects is required to determine the most suitable simulation facility for each biological object chosen. The quality of a simulation method is highly dependent on parameters like organism size, gravity perception time, threshold values and type and dense of culture media (Briegleb 1992; Hoson et al. 1997; Klaus et al. 1998; Hammond and Allen 2011).
In the past decades, 3D clinostats and the RPM operated in the $3 \mathrm{D}$ real random mode have been favored by investigators assuming that two rotation axes provide options for more complex ways to average the sensing of the gravity vector, thus, simulating $\mu g$ more perfectly. To critically assess this assumption, the tip-growing unicellular rhizoids of the green algae Chara were chosen to demonstrate and analyze in detail the effects of the different modes and different rotational velocities on the dynamics of the actin-dependent basipetal movements of statoliths after abolishing the gravity force.

The Chara rhizoid is one of the best studied cell model for research on plant gravity sensing and the processes of gravity-oriented growth (Kiss 1997; Braun and Limbach 2006; Häder et al. 2017). In tip-downward growing rhizoids, statoliths are kept in a dynamically stable position 10 $25 \mu \mathrm{m}$ above the cell tip by two counteracting forces. The gravitational force $(1 \mathrm{~g})$ pulling the statoliths toward the cell tip is exactly counteracted by cytoskeletal forces generated by the complexly organized actomyosin system (Braun et al. 2002). Any deviation from the vertical growth direction, however, causes statoliths to sediment onto a specific gravisensitive area of the subapical cell flank, thereby initiating the gravitropic curvature response. Abolishing the gravity force results in an axial displacement of statoliths away from the cell tip (Volkmann et al. 1991; Buchen et al. 1993; Braun et al. 2002). Data on the dynamics of this movement of statoliths is available from various real $\mu g$ conditions like parabolic plane flights, sounding rocket missions (TEXUS, MAXUS) and several space shuttle missions (Bunchen et al. 1991, 1993; Volkmann et al. 1991; Braun et al. 2002; Limbach et al. 2005). Chara rhizoids which have already been studied on 2Dand 3D clinostats (Cai et al. 1997; Hoson et al. 1997; Braun et al. 2002) showed similar effects like in real microgravity.

The present study aims to carefully evaluate the microgravity simulation quality of the different RPM modes by analyzing the basipetal statoliths transport in rhizoids particularly focusing on the relevance of a second rotation axes on the gravity-sensitive statoliths-cytoskeleton based system in this well-understood Chara rhizoid model system.

\section{Material and Methods}

\section{Rhizoid Culture}

Chara globularis Thuill. was collected from a pond in the botanical garden in Bonn. For rhizoid cultivation, thallus segments containing two nodes and one internode were cut and the side branches were removed to induce 
rhizoid development. Thallus segments were embedded in $1.2 \%$ agar (Sigma-Aldrich, Missouri, USA) on microscopic slides, covered with long cover glasses and stored in staining jars under a $60 \mathrm{~W}$ tungsten light bulb at a photon fluence rate of approx. $60 \mu \mathrm{mol}^{*} \mathrm{~m}^{-2 *} \mathrm{~s}^{-1}$. After 5 days, the rhizoids developed and were used at a length of $5-10 \mathrm{~mm}$ for clinorotation experiments and for the MAXUS microgravity experiments.

\section{Random Positioning Machine (RPM)}

The "Desktop RPM" from Dutch Space (Leiden, Netherlands) was used to subject characean rhizoids to simulated microgravity conditions (Fig. 1). Its two frames are positioned perpendicular to each other and can be rotated separately. In 2D mode, the software-supported motorcontrolling runs only one of the two frames constantly around one horizontal axis (Fig. 1a). In 3D real random mode, the sample mounted on the inner frame is rotated around two axes with randomly changing rotational speed and directions. In this mode only the speed range is specified by the operator. For the present study, the RPM was operated in the 2D clinorotation mode with $3 \mathrm{rpm}$ and 60 $\mathrm{rpm}$ as well as in the 3D real random mode with $2-11 \mathrm{rpm}$, $30-60 \mathrm{rpm}$ and 60-85 rpm.
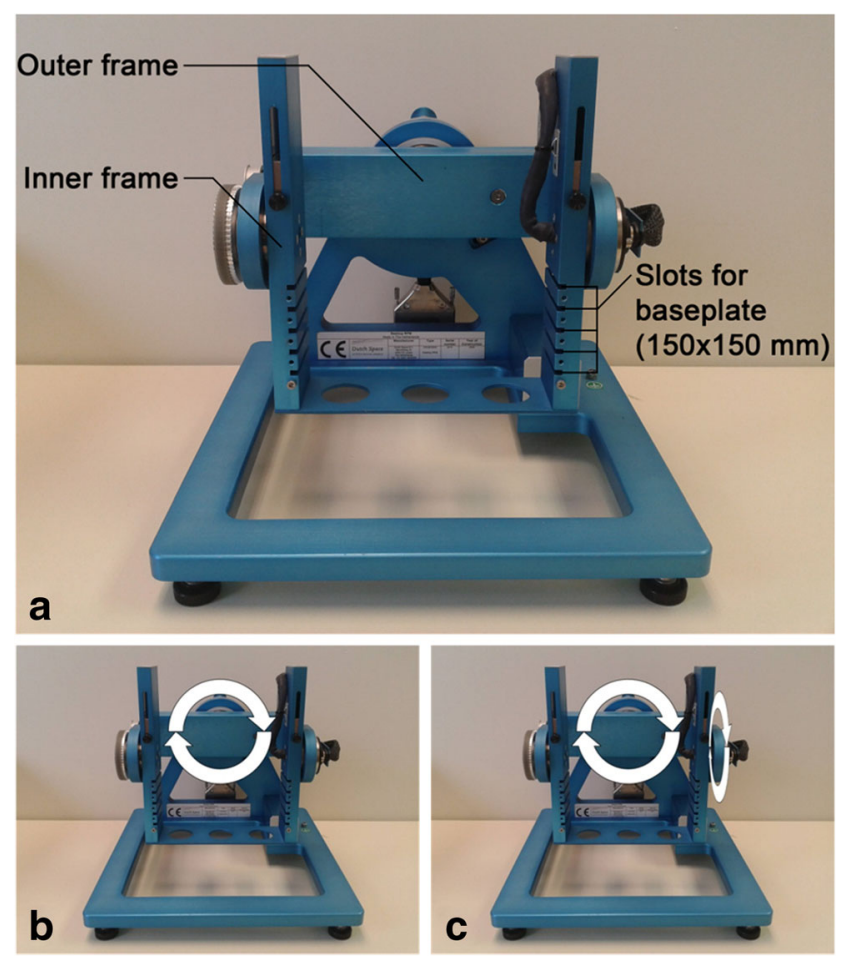

Fig. 1 Desktop Random Positioning Machine RPM (a). In 2D clinorotation mode either the inner or the outer frame rotates constantly around one horizontal axis (b). In 3D real random mode both frames rotate with random speeds and directions (c)
Chara rhizoids were mounted on the stage of one of the two platforms in such way that the cell tip was precisely aligned to the rotations axis in case of the $2 \mathrm{D}$ mode. In the 3D mode, the rhizoid tip was precisely positioned in the center of the two axes. All experiments were performed at $22+/-1{ }^{\circ} \mathrm{C}$.

For videorecording, a CMOS camera module (RSOV7949-1818, Conrad Electronic, Hirschau Germany), a 32x objective (Zeiss LD ACHROPLAN Ph2, 32× $>0.40$; $\infty / 1.5$, Carl Zeiss AG, Oberkochen, Germany) and a white LED (75, 14000 mcd, Conrad Electronic, Hirschau, Germany) for illumination were integrated into optomechanical system components (Microbench system, Qioptiq, Paris, France).

Statolith tracking was done by labeling the lower and upper border of the statoliths complex in a sequence of still images prepared from the AVI-video sequences. The distances of the lower and upper border of the statoliths complex to the cell tip was measured with ImageJ (version1.44p, Wayne Rasband, National Institutes of Health, Bethesda, USA).

\section{MAXUS-Sounding Rocket Flights}

Characean rhizoids were launched on ESA-funded MAXUS sounding rockets from Esrange (European Space and Sounding Rocket Range) near Kiruna 200 km North off the Arctic Circle in northern Sweden (MAXUS-8 mission). The MAXUS rocket is a non-spinning rocket that uses a rate control system to stabilize the payload. With peak accelerations of up to $13 \mathrm{~g}$, the single-stage solid-fuel rocket motor propels the payload above the Earth atmosphere and $86 \mathrm{~s}$ after burn-out and motor separation, the parabolic flight maneuver provides microgravity conditions in a quality of $<10^{-4} \mathrm{~g}$ for approximately $840 \mathrm{~s}$ reaching an apogee of approximately $705 \mathrm{~km}$. Thereafter, reentry of the payload terminates the microgravity phase, the parachute opens and the payload lands usually $50-75 \mathrm{~km}$ north of Esrange in an uninhabited area.

The microscope experiment module flown on MAXUS was accommodated within a $640 \mathrm{~mm}$ diameter cylindrical payload element on a platform structure housing the power supply and electronics in the lower part and the microscope and the late-access unit in the upper part. The biological samples were embedded in $1.2 \%$ agar in vacuum-tight growth chambers and mounted in a late access unit, which allowed the insertion of the samples into the payload 90120 min before launch.

A telemetry system multiplexes and transmits experiment and housekeeping data from the experiment modules and the in-flight service systems to the ground. Microscope operations during flight were executed form a ground terminal in the control room via telecommand channels. 


\section{Video Analysis}

Statolith tracking and recording the position of the statoliths complex during clinorotation and the MAXUS flight was done by outlining the statoliths complex in a sequence of images prepared from AVI-video sequences. The distance between the upper and the lower border of the statoliths complex from the cell tip was measured with ImageJ, Wayne Rasband, National Institutes of Health, Bethesda, USA.

\section{Vibration Measurements}

Vibrations were measured with a plane piezo electrical transducer mounted on the inner or outer frame to detect vibrations in the range of $0-25 \mathrm{kHz}$. Detected signals were recorded via a PC Soundcard and WaveLab 4.0 (Steinberg) and analyzed with Matlab to visualize the frequency spectrum of the measured vibrations.

\section{Results}

Under $1 g$ conditions, statoliths in tip-downward growing rhizoids form a disk-like dense complex located in a dynamically stable position above the cell tip. During the $840 \mathrm{~s}$ period of high quality microgravity (typically $10^{-4} \mathrm{~g}$ ) provided by the MAXUS-8 sounding rocket flight, the statoliths became more loosely arranged and were displaced away from the tip toward the subapical area of the cell. The distance of the lower border of the statoliths complex increased up to $23.22 \mu \mathrm{m}$, the distance of the upper border increased up to $46.13 \mu \mathrm{m}$ from the tip at the end of the 840s-period of microgravity, before the microgravity period was terminated by vibrations generated by the reentry of the payload module into the Earth's atmosphere.

The kinetic data of statoliths displacement of four Chara rhizoids during the $840 \mathrm{~s}$ period of real-microgravity conditions of a MAXUS-flight was taken as reference for evaluating the quality of microgravity simulation provided by a Random-Positioning Machine running with one axis (2D mode) or two axes (3D mode) and different rotational speeds (2D), speed ranges and directions (3D). All rhizoids which were clinorotated for $20 \mathrm{~min}$ in the different modes ( $\mathrm{n}=11$ each) continued to grow straight without showing any curvature response which indicates that sedimentation of statoliths triggering a gravitropic growth correction did not occur during any of the clinostat treatments. Like in real microgravity, the statoliths complex was rearranged from a flat disk-like shape into an axially elongated shape further away from the cell tip.

\section{D Clinorotation}

When rhizoids were clinorotated on the RPM (Fig. 1) in 2D mode (using one axis), only the inner frame was used. Control experiments on the outer frame did not reveal any significant differences in the kinetics of statoliths displacement (data not shown). Slow clinorotation with $3 \mathrm{rpm}$ in 2D mode resulted in the increase of the distance of the lower and the upper border of the statoliths complex from $8.12 \mu \mathrm{m}$ and $18.95 \mu \mathrm{m}$ up to $22.53 \mu \mathrm{m}$ and $39.27 \mu \mathrm{m}$ after $840 \mathrm{~s}$ and $26.21 \mu \mathrm{m}$ and $42.94 \mu \mathrm{m}$ after $20 \mathrm{~min}$ of clinorotation, respectively. The statoliths at the upper border of the complex reached only $84.9 \%$ of the distance compared to real microgravity (MAXUS experiments). The statoliths were considerably more dispersed over the rhizoid diameter and formed a more loosely arranged complex under slow clinorotation than in real-microgravity conditions (Fig. 3a).

Increasing the rotational speed of the single axis to $60 \mathrm{rpm}$ strongly promoted the basipetal displacement of statoliths (Fig. 3b). The distances of the lower border and the upper border of the statoliths complex increased up to $25.27 \mu \mathrm{m}$ and $46.76 \mu \mathrm{m}$ after $840 \mathrm{~s}$ and up to $29.92 \mu \mathrm{m}$ and $51.68 \mu \mathrm{m}$ after $20 \mathrm{~min}$ of clinorotation, respectively. Thus, the position of the uppermost statoliths was almost identical $(101.3 \%)$ to the results from the MAXUS real-microgravity experiment and the shape of the complex was also similar to the relatively dense assembly of statoliths in rhizoids und real microgravity conditions.

\section{D Clinorotation}

In the real random mode, both frames rotated with rotational speeds in a range of $2-11 \mathrm{rpm}, 30-60 \mathrm{rpm}$ or 60 $85 \mathrm{rpm}$. Statoliths in rhizoids clinorotated at $2-11 \mathrm{rpm}$ were displaced up to $22.42 \mu \mathrm{m}$ (lower border) and 38.02 (upper border) after $840 \mathrm{~s}$ and up to $25.12 \mu \mathrm{m}$ and $41.30 \mu \mathrm{m}$ after 20 min, respectively (Fig. 2c). In comparison with the MAXUS data, the statoliths reached a maximal distance of only about $82.4 \%$ of the real-microgravity reference. The statoliths were distributed over a relatively large area forming a rather irregularly outlined complex (Fig. 3).

Increasing the range of rotational speed to $30-60 \mathrm{rpm}$ resulted in a basipetal displacement of statoliths up to $20.98 \mu \mathrm{m}$ (lower border) and $40.13 \mu \mathrm{m}$ (upper border) after $840 \mathrm{~s}$ and $24.18 \mu \mathrm{m}$ (lower border) and $42.84 \mu \mathrm{m}$ (upper border) after $20 \mathrm{~min}$, respectively, which represents only $9.0 \%$ of the real-microgravity reference value (Fig. $2 \mathrm{~d}$ ).

At a range of $60-85 \mathrm{rpm}$ rotational speed, statoliths were displaced up to $25.92 \mu \mathrm{m}$ and $42.45 \mu \mathrm{m}$ after $13 \mathrm{~min}$ and $29.59 \mu \mathrm{m}$ and $45.16 \mu \mathrm{m}$ after $20 \mathrm{~min}$, 

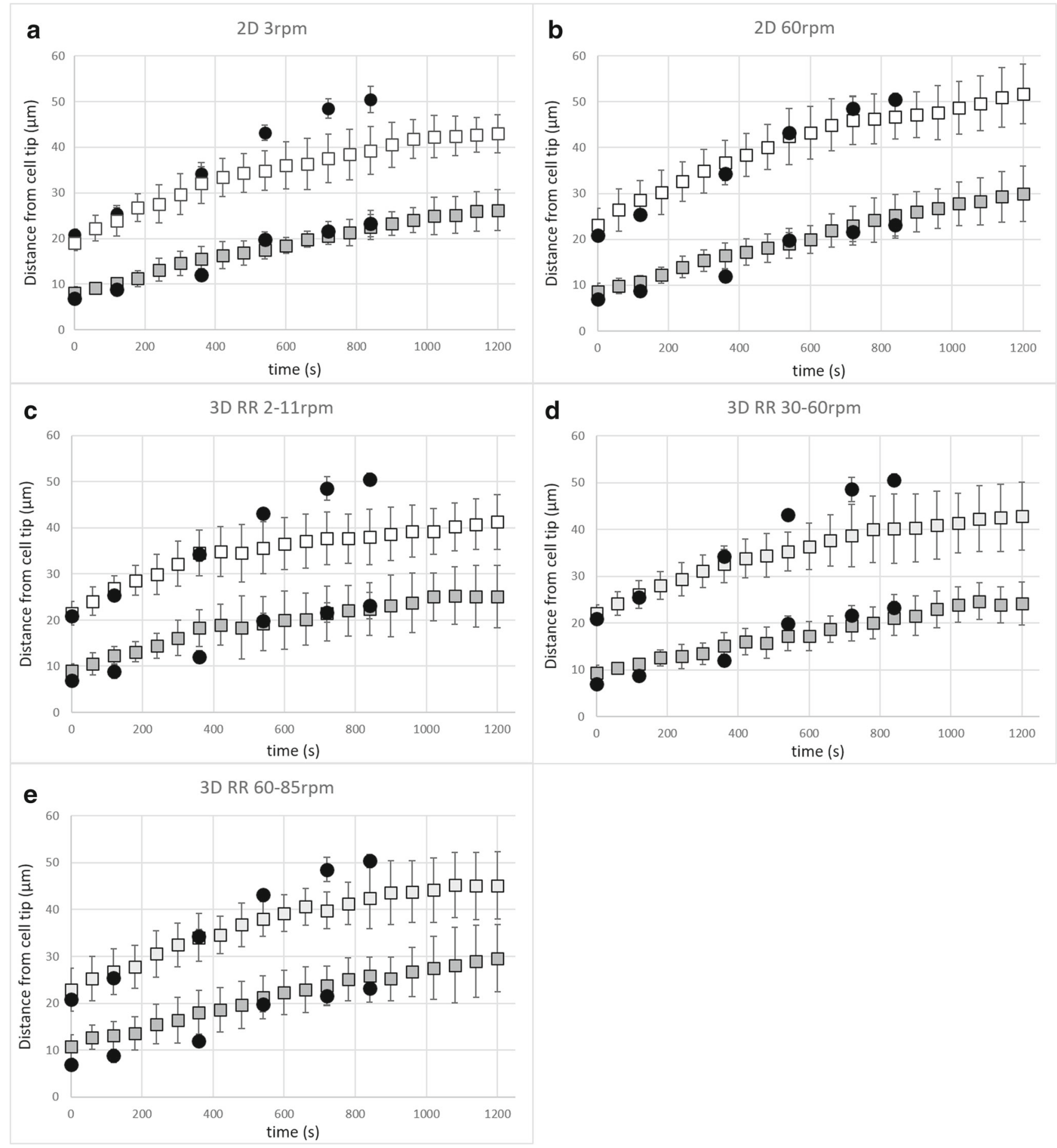

Fig. 2 Graphs showing the average distances of the lower (gray squares) and upper border (white squares) of the statolith complex in rhizoids $(n=11)$ clinorotated for $20 \mathrm{~min}$ in $2 \mathrm{D}$ mode (rotating one frame; see Fig. 1a) with $3 \mathrm{rpm}$ (a) and $60 \mathrm{rpm}(\mathbf{b})$ and in 3D realrandom mode (RR; rotating both frames; see Fig. 1c) with 2-11 rpm

respectively (Fig. 2e). Thus, statoliths reached approx. $86.9 \%$ of the real-microgravity reference value. Statoliths always show trembling movements in all directions also in

(c), 30-60 rpm (d) and 60-85 rpm (e). Black circles indicate the lower and upper border of statolith complexes in rhizoids $(n=4)$ in real-microgravity conditions during the MAXUS-8 sounding rocket flight

their resting position at $1 g$ as well as during net-basipetal displacement in real-microgravity and in 2D-clinorotated rhizoids. However, trembling motion was considerably 


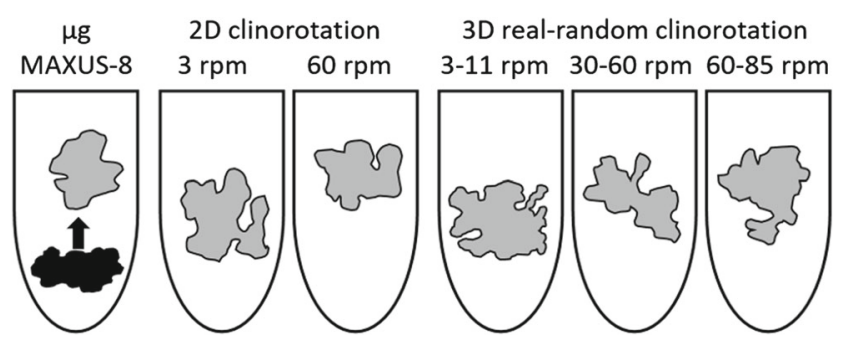

Fig. 3 Outlines of statolith complexes in representative rhizoids at $1 \mathrm{~g}$ (black area) and after 13 min of clinorotation in the 2D and 3D modes in comparison to real microgravity ( $\mu g$, MAXUS-8 flight)

more pronounced during 3D-clinorotation (data not shown). Furthermore, the statoliths complex was considerably less densely packed during 3D-clinorotation than during 2D clinorotation and in real-microgravity (Fig. 3).

\section{Vibration Analysis}

For measuring vibration pattern the samples experienced during $2 \mathrm{D}$ and $3 \mathrm{D}$ operation, the sensor was fixed next to the sample on the inner frame of the RPM. Fast 2D clinorotation at $60 \mathrm{rpm}$ performed with the inner frame of the RPM caused only moderate vibrations in the range of $0-10 \mathrm{kHz}$ (Fig. 4a). In contrast to the 2D mode, 3D clinorotation in real random mode with $60-85 \mathrm{rpm}$ caused much stronger and unregular vibration patterns and considerably higher relative amplitudes over the whole measured frequency spectrum between $0-25 \mathrm{kHz}$ (Fig. 4b).

\section{Discussion}

Characean rhizoids were chosen to evaluate the microgravity-simulation quality of the different 2D- and $3 \mathrm{D}$-operation modes of the RPM due to the fact that the

a

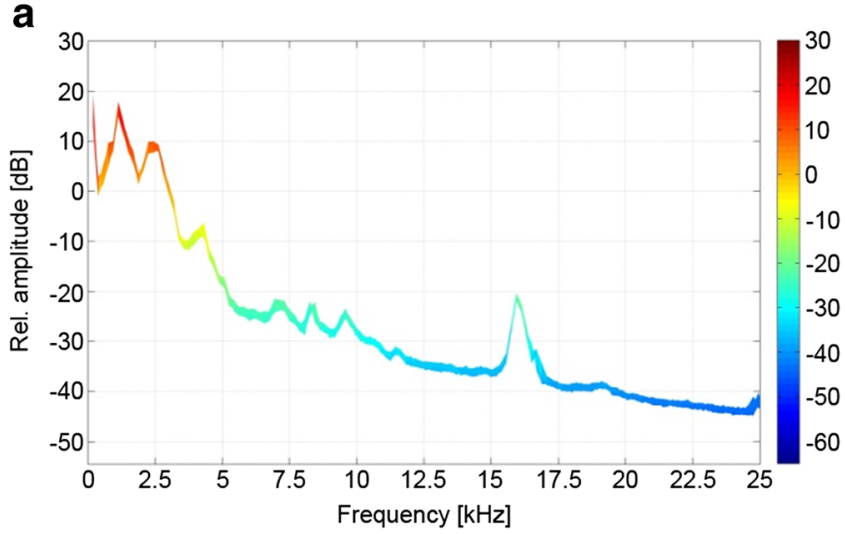

Fig. 4 Frequency spectrum during fast 2D clinorotation with $60 \mathrm{rpm}$ performed with the inner frame (a) and 3D clinorotation in real random mode with 60-85 rpm (c). Relative amplitudes of vibrations were position of the numerous statoliths in the subapical region of rhizoids is precisely controlled by the interaction of actomyosin and gravitational forces (Braun et al. 2002). Only in high-quality real microgravity (weightlessness or free fall situation), the basipetal transport of statoliths toward a new equilibrium position further away from the cell tip is exclusively controlled by the actomyosin system. Any remaining accelerations or vibrations inevitably interfere with the transport process and result in impaired transport kinetics and a more dispersed arrangement of statoliths over the cell diameter. This effect has been reported in slow-rotating clinostat studies e.g. by Cai et al. (1997) and in 3D-clinostat studies by Hoson et al. (1997).

In order to evaluate the $\mu g$-simulation quality of the different RPM modes, in this study, the kinetics of the basipetal transport of statoliths was analyzed during the initial 20 min of RPM-operation in the different 2D and 3D modes and compared to the kinetics of the statoliths transport during the 13-min real-microgravity phase of MAXUS-sounding rocket flights. The results show that the different velocities of rotation as well as the $2 \mathrm{D}$ and $3 \mathrm{D}$ mode have considerable and a very distinctive impact on the kinetics of the statoliths transport.

There is convincing evidence that the $\mu g$-simulation quality positively correlates with the rotational speed of the RPM. Fast 2D and 3D clinorotation with rotational velocities of $60 \mathrm{rpm}$ and higher caused a displacement of statoliths whose kinetics was very similar to those observed under real- $\mu g$ conditions of MAXUS sounding rocket flights. Slower clinorotation impaired the basipetal transport and lead to more dispersed statoliths. Earlier studies have shown that more dispersed statoliths sediment more slowly upon gravistimulation, e.g. when the rhizoid is tilted into a horizontal position at $1 g$ leading to a delayed graviresponse in the form of gravitropic curvature which brings the cell's tip back into the tip-downward orientation within a few

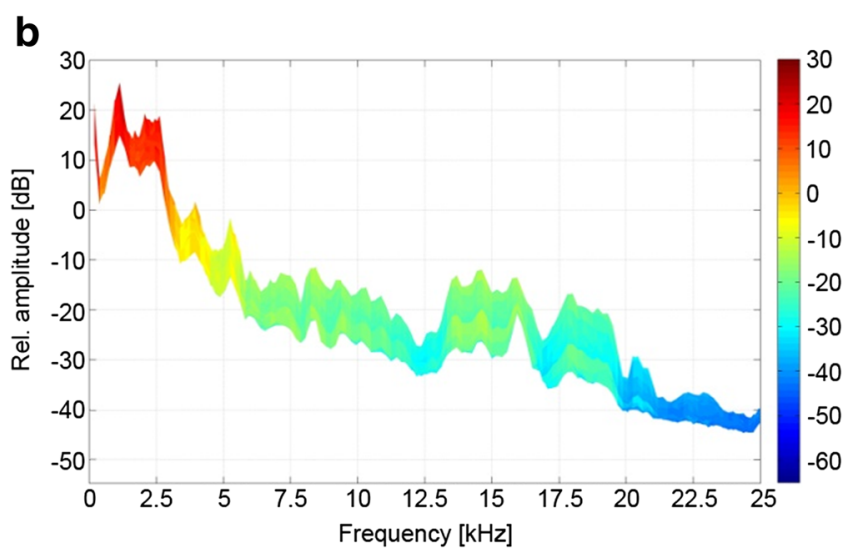

measured in a range of $0-25 \mathrm{kHz}$. The intensity of the relative amplitude between -60 (dark blue) and $30 \mathrm{~dB}$ (dark red) is color coded (color bar) 
hours (Herranz et al. 2013). A similar effect was observed after centrifugation of rhizoids which also led to a disturbed sedimentation process most like caused by an adaptation of the actomyosin system (Braun and Sievers 1993).

The best results, namely, a situation closely resembling the basipetal transport of statoliths in real- $\mu g$, were achieved with rhizoids rotating precisely along a single axis with a rotational speed of $60 \mathrm{rpm}$. Using the second axis did not improve the statoliths transport kinetics, however, resulted in generally more loosely arranged statoliths complexes in slower and faster rotated rhizoids. The vibration sensor measurements clearly suggest an overall higher level and irregularity of the vibration amplitudes to be the main causes for the negative influence of a second rotating axis on the statoliths transport process. The erratic vibration pattern of fast 3D-clinorotation appears to result from the constantly changing rotation direction as well as the constantly varying rotational speed.

These results are consistent with reports of RPM experiments with organisms like free-swimming protists like Euglena und Paramecium as well as Drosophila, fish and some human cell types (Herranz et al. 2013). When the RPM is operated in the real-random mode, microgravity simulation is characterized by a constantly changing rotation velocity and in addition also a constantly changing direction of rotation. Clear evidence for the negative impact of the real-random mode on cellular activities comes from experiments with the fast and sensitive shear stress reporter system of dinoflagellates (Hauslage et al. 2017). Shear stress and hydrodynamic gradients trigger a luciferin/luciferase reaction in dinoflagellates probably via a calcium mediated signaling cascade (Mallipattu et al. 2002; Jin et al. 2013; Von Dassow and Latz 2002). Bioluminescence is part of the defense mechanism of these dinoflagellates. The combination of random speed and direction of the PRM had a stronger impact on bioluminescence of the dinoflagellate Dinocystis noctiluca than only changing the rotational speed or using a fast and constantly rotating clinostat at $60 \mathrm{rpm}$ (Hauslage et al. 2017) clearly indicating a higher stress level caused by the 3D operation mode.

The advantage of the fast 2D mode, the lower level of vibrations and the generally better microgravity quality, however, certainly applies only for small objects like dinoflagellates and characean rhizoids as long as they are perfectly aligned along the rotation axis. Good quality microgravity is provided only in a range of a few millimeters along the axis. With increasing speed, the radius around the rotation axis strongly decreases in which good-quality microgravity is provided. However, in contrast to slower rotational speeds $(2-11 \mathrm{rpm})$ which randomize the gravity vector without effectively preventing sedimentation, from a purely technical perspective, higher rotation speeds (>60 rpm) provide near weightlessness due to the almost complete prevention of any sedimentation process (Briegleb 1992).

Depending on the sensitivity (namely perception and response time of a specific process or organism), slower 2D and 3D clinorotation were applied for investigations on physiological processes like proliferation/growth and development in bigger organisms like Arabidopsis plants (Herranz et al. 2013). At higher rotational speeds, residual $g$-forces or even centrifugal forces with increasing distance from a fast rotating axis, induce shear forces and might cause stress symptoms in the organisms. The experiments presented in this paper demonstrate that ground-based facilities have to be carefully evaluated for their nongravitational effects on specific organisms and, also, that high-quality microgravity simulation can be achieved supplementing rare and expensive real-microgravity studies in low Earth orbit.

For small objects like the tip of a Chara rhizoids (embedded in agar), where all processes of the gravitropic signaling pathways occur in a very small area, fast clinorotation in 2D and 3D mode provides a good simulated microgravity environment as long as the tip is perfectly oriented in the center of rotation. However, the lower stress and vibration levels make 2D clinorotation the better choice for investigating gravitropic signaling and the gravitropic response in characean rhizoids.

Open Access This article is distributed under the terms of the Creative Commons Attribution 4.0 International License (http:// creativecommons.org/licenses/by/4.0/), which permits unrestricted use, distribution, and reproduction in any medium, provided you give appropriate credit to the original author(s) and the source, provide a link to the Creative Commons license, and indicate if changes were made.

\section{References}

Borst, A.G., van Loon, J.J.W.S.: Technology and developments for the random positioning machine, RPM. Microgravity Sci. Technol. 21, 287-292 (2009)

Braun, M., Sievers, A.: Centrifugation causes adaptation of microfilaments Studies on the transport of statoliths in gravity sensing Chara rhizoids. Protoplasma 174, 50-61 (1993)

Braun, M., Limbach, C.: Rhizoids and protonemata of characean algae: model cells for research on polarized growth and plant gravity sensing. Protoplasma 229, 133-142 (2006)

Braun, M., Buchen, B., Sievers, A.: Actomyosin-mediated statolith positioning in gravisensing plant cells studied in microgravity. J. Plant Growth Regul. 21, 137-145 (2002)

Briegleb, W.: Some qualitative and quantitative aspects of the fastrotating clinostat as a research tool. ASGSB Bull. 5, 23-30 (1992)

Buchen, B., Hejnowicz, Z., Braun, M., Sievers, A.: Cytoplasmic streaming in Chara rhizoids. Protoplasma 165, 121-126 (1991) 
Buchen, B., Braun, M., Hejnowicz, Z., Sievers, A.: Statoliths pull in microfilaments - Experiments under microgravity. Protoplasma 172, 38-42 (1993)

Brungs, S., Egli, M., Wuest, S., Christianen, P.C.M., van Loon, J.J.W.A., Ngo-Anh, J., Hemmersbach R.: Facilities for simulation of microgravity in the ESA ground-based facility programme. Microgravity Sci. Technol. ISSN 0938-0108 (2016). https://doi.org/10.1007/s12217-015-9471-8

Cai, W.M., Braun, M., Sievers, A.: Displacement of statoliths in Chara rhizoids during horizontal rotation on clinostats. Acta Biol. Exp. Sinica 30, 147-155 (1997)

Häder, D.-P., Braun, M., Grimm, D., Hemmersbach, R.: Gravireceptors in eukaryotes - a comparison of case studies on the cellular level. npj Microgravity 3, 13 (2017). https://doi.org/10.1038/s41526-017-0018-8

Hammond, T., Allen, P.: The Bonn criteria: minimal experimental parameter reporting for clinostat and random positioning machine experiments with cells and tissues. Microgravity Sci. Technol. 23, 271-275 (2011)

Hauslage, J., Cevik, V., Hemmersbach, R.: Pyrocystis noctiluca represents an excellent bioassay for shear forces induced in ground-based microgravity simulators (clinostat and random positioning machine). npj Microgravity 3, 12 (2017). https://doi.org/10.1038/s41526-017-0016-X

Herranz, P., Anken, R., Boonstra, J., Braun, M., Christianen, P.C., de Geest, M., Hauslage, J., Hilbig, R., Hill, R.J.A., Lebert, M., Medina, F.M., Vagt, N., Ullrich, O., van Loon, J.J.W.A., Hemmersbach, R.: Groundbased facilities for simulation of microgravity: organism-specific recommendations for their use, and recommended terminology. Astrobiology 13(1), 1-17 (2013). https://doi.org/10.1089/ast.2012.0876
Hoson, T., Kamisaka, S., Masuda, Y., Yamashita, M., Buchen, B.: Evaluation of the three-dimensional clinostat as a simulator of weightlessness. Planta 203, 187-197 (1997)

Kiss, J.Z.: Gravitropism in the rhizoids of the alga Chara: a model system for microgravity research. Biol. Bull. 192, 134-136 (1997)

Jin, K., Klima, J.C., Deane, G., Dale-Stokes, M., Latz, M.I.: Pharmacological investigation of the bioluminescence signaling pathway of the dinoflagellate Lingulodinium polyedrum: evidence for the role of stretch-activated ion channels. J. Phycol. 49, 733-745 (2013)

Klaus, D.M., Todd, P., Schatz, A.: Functional weightlessness during clinorotation of cell suspensions. Adv. Space Res. 21, 1315-1318 (1998)

Limbach, C., Hauslage, J., Schäfer, C., Braun, M.: How to activate a plant gravireceptor. Early mechanisms of gravity sensing studies in characean rhizoids during parabolic flights. Plant Physiol. 139, 1030-1040 (2005)

Mallipattu, S., Haidekker, M., Von Dassow, P., Latz, M., Frangos, J.: Evidence for shear-induced increase in membrane fluidity in the dinoflagellate Lingulodinium polyedrum. J. Comp. Physiol. 188, 409-416 (2002)

Van Loon, J.J.W.S.: Some history and use of the random positioning machine, RPM, in gravity related research. Adv. Space Res. 39, 1161-1165 (2007)

Volkmann, D., Buchen, B., Hejnowicz, Z., Tewinkel, M., Sievers, A.: Oriented movement of statoliths studied in a reduced gravitational field during parabolic flights of rockets. Planta 185, 153-161 (1991)

Von Dassow, P., Latz, M.I.: The role of $\mathrm{Ca} 2+$ in stimulated bioluminescence of the dinoflagellate Lingulodinium polyedrum. J. Exp. Biol. 205, 2971-2986 (2002) 